\title{
Network Pharmacology Study of Heat-Clearing and Detoxifying Traditional Chinese Medicine for Alzheimer's Disease
}

\author{
Hongxing Li, Xinyue Zhang $(\mathbb{D}$, Lili Gu, Ningzi Wu, Lingxi Zhang, Jiaqi Lu, and Qin Li $\mathbb{D}$
}

Key Laboratory of Neuropsychiatric Drug Research of Zhejiang Province, Institute of Materia Medica, Zhejiang Academy of Medical Sciences, Hangzhou, China

Correspondence should be addressed to Qin Li; qindai_1981@sohu.com

Received 3 November 2019; Revised 15 February 2020; Accepted 25 March 2020; Published 25 April 2020

Academic Editor: Shan-Yu Su

Copyright (C) 2020 Hongxing Li et al. This is an open access article distributed under the Creative Commons Attribution License, which permits unrestricted use, distribution, and reproduction in any medium, provided the original work is properly cited.

This study aims to explore the possible homologous mechanism of 7 frequently-used herbs for heat-clearing and detoxification in traditional Chinese medicine (HDTCM) for treating Alzheimer's disease (AD), one of the most common types of dementia, based on network pharmacology. Herbs that satisfied the criteria of containing chlorogenic acid, relating to AD and aligning with HDTCM, were simultaneously collected to determine whether they have anti-AD effect based on a survey of the literature. Herbingredient-target-disease networks were constructed by collecting information from the TCMSP and GeneCards public databases. The common targets of the herbs and AD were identified for conducting a Gene Ontology (GO) analyses and a Reactome pathway enrichment analysis. The results showed that PTGS1, IL-6, CASP3, and VEGFA were the predicted key gene targets. The IL-4 and IL-13 signaling pathway, the ESR-mediated signaling pathway, and the extranuclear estrogen signaling pathway were the significant pathways associated with the 7 herbs. This study revealed that the analogous anti-AD mechanism of the 7 herbs of HDTCM may be associated with anti-inflammation, which is a common effect of the chlorogenic acid and quercetin components.

\section{Introduction}

Alzheimer's disease (AD), one of the most common neurodegenerative diseases in people over 65 years old, is characterized by neurofibrillary tangles, senile plaques, neuronal loss, and cognitive decline [1-3]. According to a report, the number of people diagnosed with $\mathrm{AD}$ will be no less than 130 million by 2050 [4]. However, the mechanism of $\mathrm{AD}$ remains unclear. More than 200 clinical trials for $\mathrm{AD}$ around the world have been terminated because of ineffective treatment, and of the few remaining treatments, none can completely prevent the progression of $\mathrm{AD}$ [5]. Thus, searching for new $\mathrm{AD}$ drug resources, such as traditional Chinese medicine (TCM) and natural products, is of particular importance [6].

TCM, especially the herbs and formulae, has been used for more than a few thousand years in China and other Southeast Asian countries to prevent or cure all kinds of diseases, including neurodegenerative diseases $[7,8]$. Based on TCM theory, ameliorating the syndrome of hyperactivity of heart-liver fire, a typical syndrome of $\mathrm{AD}$, by using heatclearing and detoxifying traditional Chinese medicine (HDTCM) is beneficial to retard the pathological progression of AD. For example, Huanglian-Jie-Du-Tang, a decoction that is composed of HDTCM relevant herbs such as Coptis chinensis, Cortex Phellodendri, and Scutellaria baicalensis, is used for clearing heat, purging pathogenic fire, and reducing amyloid- $\beta$ accumulation during the treatment of $\mathrm{AD}$ [9]. In addition, a large number of modern pharmacological studies on natural ingredients isolated from HDTCM sources (such as geniposide [10], andrographolide [11], and berberine [12]) and their effects on AD have been performed. Therefore, developing new natural medicines based on HDTCM brings hope to AD patients.

Chlorogenic acid (CGA), a polyphenol component, is a widely available component in sources of HDTCM such as Lonicera japonica [13]. The CGA content is used as a quality control standard for some other herbs of HDTCM and TCM formulae, such as Yinzhi detoxifying granules and Qingrejiedu oral liquid, in China. More importantly, CGA can 
significantly alleviate inflammation and oxidative stress, two important promoters of $\mathrm{AD}$. In addition, some reports have indicated that CGA has potential neuroprotective effects. Therefore, CGA was selected as one of the main components used for screening candidate herbs.

In this study, 7 herbs were screened for further research. Currently, research on these herbs in $\mathrm{AD}$ treatment is limited to studies on monomer components or extracts. Network pharmacology $[14,15]$ is used to systematically evaluate the pharmacological effects of drugs with multiple components and multiple targets by establishing links between targets, drugs, and diseases based on the principles of systems biology. Given the characteristics of TCM and shortcomings of obsolete TCM research methods, network pharmacology was performed to explore the possible analogous mechanisms of the 7 herbs of HDTCM on AD. The workflow of this study is described in Figure 1.

\section{Materials and Methods}

2.1. Identification of Candidate Herbs. Herbs that satisfied the following criteria were collected, respectively: (i) contained CGA; (ii) related to $\mathrm{AD}$; and (iii) aligned with HDTCM. The herbs containing CGA were collected from the Traditional Chinese Medicine Systems Pharmacology database [16] (TCMSP, http://lsp.nwu.edu.cn/tcmsp.php). The herbs relevant to $\mathrm{AD}$ were also collected from the TCMSP database. The classification criteria of HDTCM were based on Chinese Pharmacopoeia 2015 (part I). All the candidate herbs were analyzed with Venny 2.1.0 (https:// bioinfogp.cnb.csic.es/tools/venny/index.html) to determine the herbs that met the above criteria simultaneously. Finally, the literature was reviewed to identify the herbs have antiAD pharmacodynamic effects.

2.2. Main Active Ingredient Screening and Target Collection. It was confirmed that the ADME (absorption, distribution, metabolism, and excretion) screening model is faster and more effective than other traditional models [17-19]. Therefore, the main active ingredients of these herbs were screened in the TCMSP database on the basis of two pharmacokinetic parameters: oral bioavailability (OB) and drug-likeness (DL). OB is extensively used to evaluate the ability of drugs to overcome absorption barriers and enter the blood circulation system and is determined by calculating the relationship between the drug and cytochrome P450s and P-glycoprotein $[17,20]$. DL represents the ability of potential ingredients to become effective drugs by calculating the similarity with a known drug, which is beneficial to optimize pharmacokinetic properties to affect ADME $[17,21]$. The active ingredients were considered viable according to ADME features with $\mathrm{OB} \geq 30 \%$ and $\mathrm{DL} \geq 0.18$, two critical value that indicate acceptable oral bioavailability and drug-likeness as previously described [22]. All targets (including the validated and predicted targets) related to these active ingredients were extracted from the TCMSP database and entered into the UniProt database [23] (http:// www.uniprot.org/) to obtain target-relevant gene names.
2.3. Target Fishing for AD Gene Targets. GeneCards is a comprehensive compendium of annotative information about human genes [24]. Significant AD-related genes were mainly collected from GeneCards (https://www. genecards.org/) based on a relevance score $>10$, a threshold that indicates a significantly high correlation with AD.

2.4. Network Construction and Analyses. In this study, network pharmacology was used to explore the interrelationships of the herbs, their ingredients, and targets with $\mathrm{AD}$, which were represented by nodes and edges. The common targets of $\mathrm{AD}$ and the main active ingredients of the 7 herbs were, respectively, determined by $R$, a free software for statistical computing and graphic visualization. The shared targets for at least 5 herbs and $\mathrm{AD}$ were also processed by $\mathrm{R}$, and the results were added to STRING [25] (https://string-db.org/) to construct a protein-protein-interaction (PPI) network. To comprehensively study the potential mechanism of the 7 kinds of herbs on $\mathrm{AD}$, the herb-ingredient-target-disease networks and the network of herb-AD common targets were constructed by using Cytoscape 3.7.1 [26].

2.5. Gene Ontology and Pathway Enrichment Analyses. Gene Ontology [27] (GO), a widespread and comprehensive computational model, provides gene annotations and a logical framework of gene functions. OmicShare Tools (https://www.omicshare.com/) was utilized to perform GO enrichment analyses and thus reveal the functional changes in these targets in three respects: molecular biological function (genes that regulate molecular activity), biological process (biological programs), and cellular components (the relationship between cellular structure and gene function) [27]. Reactome [28] (http://reactome.ncpsb.org/), a visualization pathway database that highlights shared parent-child relationships among pathways to reveal underlying functional processes, was used to determine the potentially enriched pathways involved in the anti-AD effect of the 7 herbs.

\section{Results}

3.1. Candidate Herb Information. 61 herbs containing CGA and 499 herbs related to AD were collected from the TCMSP database. Furthermore, 96 herbs for which heat-clearing and detoxification effects had been clearly identified were found in the Chinese Pharmacopoeia 2015 (part I). Then, 12 herbs that met the above criteria simultaneously emerged (Table S1). Further research based on the literature review revealed that only seven out of these twelve herbs had an anti-AD pharmacodynamic effect (Table 1). Ultimately, as shown in Figure 2, 7 herbs, namely, Andrographis paniculata (AP), Coptis chinensis (CC), Cortex Phellodendri amurensis (CPA), Lonicera japonica (LJ), Houttuynia cordata (HC), Centella asiatica (CA), and Gardenia jasminoides (GJ), were chosen for further study. 


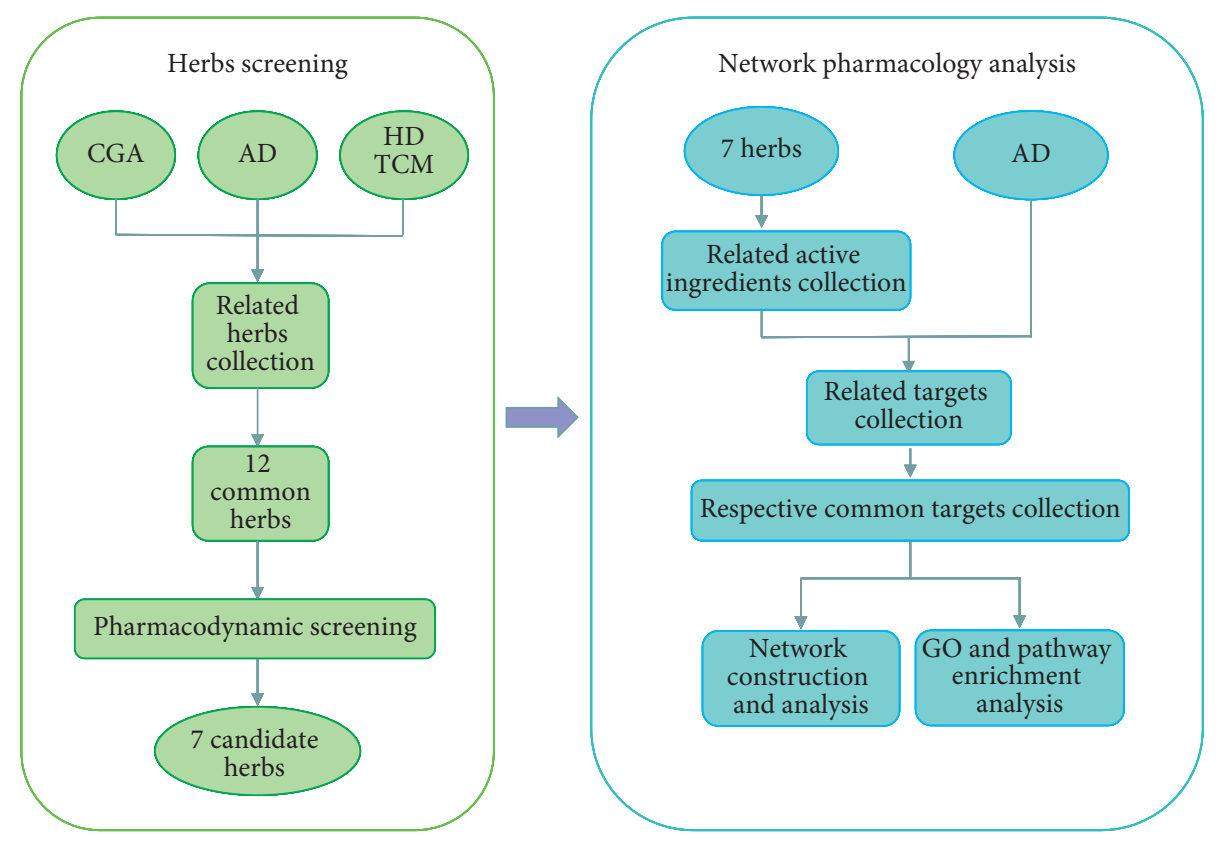

FIGURE 1: Workflow of this study.

TABle 1: Pharmacodynamic effect of anti-AD of 7 herbs.

\begin{tabular}{|c|c|c|}
\hline Latin name & Chinese name & Pharmacodynamic effect of anti-AD \\
\hline Andrographis paniculata & Chuanxinlian & $\begin{array}{r}\text { Andrographolide, one of main active component of AP, reduced or elim } \\
\text { and attenuated } \mathrm{A} \beta \text { neurotoxicity }[11,29,30]\end{array}$ \\
\hline Coptis chinensis & Huanglian & $\begin{array}{l}\text { Berberine and polysaccharide from } \mathrm{CC} \text { could reduce } \mathrm{A} \beta \text {-induced toxicity and ameliorate } \\
\text { cognitive impairment }[12,31,32]\end{array}$ \\
\hline $\begin{array}{l}\text { Cortex Phellodendri } \\
\text { amurensis }\end{array}$ & Guanhuangbai & $\begin{array}{l}\text { The ethanol extract of CPA has the potential protective effect against neurotoxicity induced by } \\
\qquad \mathrm{A} \beta[33]\end{array}$ \\
\hline iaponica & & LJ can effectively prevent the cognitive dys \\
\hline Houttuynia cordata & & $\mathrm{HC}$ water extract protects cortical neurons against A $\beta$-induced toxicity [35] \\
\hline Centella asiatica & & CA extract reduces $\mathrm{A} \beta$ level and improves neuronal health $[36,37]$ \\
\hline Gardenia jasminoides & Zhizi & GJ extract improves cognitive and memory impairment on $\mathrm{A} \beta$-induced mouse [38] \\
\hline
\end{tabular}

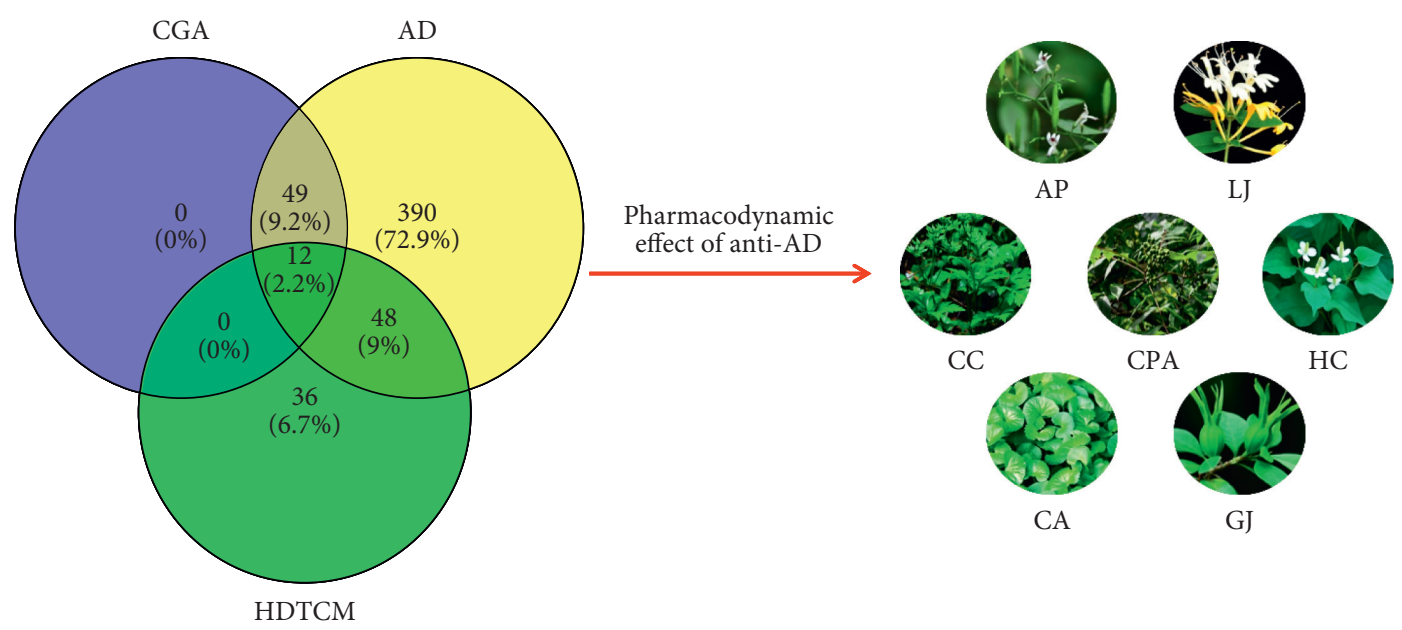

Figure 2: Screening process for 7 herbs.

3.2. Information on the Main Active Ingredients and Targets. For each of these herbs, the main active ingredient had an OB value $\geq 30 \%$ and a DL value $\geq 0.18$. Finally, a total of 64 active ingredients in these herbs were identified: 13 in AP, 10 in CC, 17 in CPA, 8 in LJ, 5 in HC, 2 in CA, and 9 in GJ. Moreover, 64 main active ingredients in the 7 herbs were 
associated with 1032 targets: 111 in AP, 222 in CC, 219 in CPA, 113 in LJ, 119 in HC, 80 in CA, and 168 in GJ (Tables S2-S8). Simultaneously, 711 significant gene targets linked to $\mathrm{AD}$ were screened out by setting the relevance score $>10$ (Table S9).

3.3. Network Construction and Analysis for the Anti-AD Targets of the 7 Herbs. First, the targets of the main active ingredients of the 7 herbs were mapped to the targets of $\mathrm{AD}$. Subsequently, the herb-ingredient-target-disease networks of the 7 herbs were constructed, respectively. Among these networks, the yellow triangle nodes represent the herbs, the green ellipse nodes represent the ingredients, the blue diamond nodes represent the targets, the red rectangle nodes represent the disease, and the edges represent the interactions between each other. Degree, one of the significant topological parameters, was used to assess the importance of an ingredient or target in the network; in other words, the higher the degree, the greater the likelihood that the herb plays a role in anti-AD effect through the active ingredient or target.

As shown in Figure 3(a), the AP network consisted of 30 nodes ( 1 herb, 1 disease, 13 ingredients, and 15 targets). This network revealed that components such as wogonin (degree $=11)$, deoxycamptothecine $($ degree $=9)$, and quercetin tetramethyl $\left(3^{\prime}, 4^{\prime}, 5,7\right)$ ether (degree $\left.=9\right)$ were the highdegree ingredients and that genes such as PTGS1 (degree $=11$ ) and AR (degree=11) were the high-degree targets. As shown in Figure 3(b), the CC network consisted of 77 nodes ( 1 herb, 1 disease, 10 ingredients, and 65 targets). This network revealed that components such as quercetin $($ degree $=58)$ and palmatine $($ degree $=9)$ were the highdegree ingredients and that genes such as PTGS2 (degree $=10)$, AR (degree $=9)$, and PTGS1 (degree =9) were the high-degree targets. As shown in Figure 3(c), the CPA network consisted of 58 nodes (1 herb, 1 disease, 17 ingredients, and 39 targets). This network revealed that components such as quercetin (degree=33), wogonin (degree $=11)$, and baicalein $($ degree $=10)$ were the highdegree ingredients and that genes such as PTGS1 $($ degree $=15), \operatorname{AR}($ degree $=11)$, and ESR1 $($ degree $=9)$ were the high-degree targets. As shown in Figure $3(\mathrm{~d})$, the LJ network consisted of 45 nodes ( 1 herb, 1 disease, 8 ingredients, and 35 targets). This network revealed that components such as quercetin (degree $=33$ ) and 5-hydroxy-7methoxy-2-(3,4,5-trimethoxyphenyl) chromone $($ degree $=7)$ were the high-degree ingredients and that genes such as PTGS1 (degree =5) and AR (degree=5) were the high-degree targets. As shown in Figure 3(e), the HC network consisted of 40 nodes (1 herb, 1 disease, 5 ingredients, and 33 targets). This network revealed that components such as quercetin $($ degree $=33)$ and kaempferol $($ degree $=15)$ were the high-degree ingredients and that genes such as NR3C2 (degree $=4$ ) and PTGS1 (degree = 3) were the highdegree targets. As shown in Figure 3(f), the CA network consisted of 36 nodes ( 1 herb, 1 disease, 2 ingredients, and 32 targets). This network revealed that component quercetin (degree $=32$ ) was the high-degree ingredients and that genes such as GSTM1 (degree =2), PTGS1 (degree=2), and IGF2 (degree $=2)$ were the high-degree targets. As shown in Figure 3(g), the GJ network consisted of 47 nodes (1 herb, 1 disease, 9 ingredients, and 36 targets). This network revealed that components such as quercetin (degree=33) and kaempferol (degree $=15$ ) were the high-degree ingredients and that genes such as PTGS1 $($ degree $=8)$ and PPARG $($ degree $=6)$ were the high-degree targets. Information about the ingredients and targets of the 7 herbs is illustrated in Table 2.

Based on the network constructs of the 7 herbs, 34 shared targets (Table S10) for at least 5 herbs and AD were further analyzed by $\mathrm{R}$ to explore analogous mechanisms of anti-AD and related biological processes among these herbs. As shown in Figure 3(h), purple represents the shared targets of 5 herbs-AD common targets (such as EGFR, ESR1, ESR2, and VEGFA), blue represents the shared targets of 6 herbsAD common targets (such as ACHE, BCL2, CASP8, and CAV1), and green represents the shared targets of 7 herbsAD common targets (such as AR, CASP3, CASP9, and F7).

3.4. PPI Network Construction and Analysis. The data from thirty-four shared targets were added to STRING to construct a PPI network for exploring the interaction relationships with each other (Figure 4(a)). The degree was calculated by NetworkAnalyzer in Cytoscape to reflect the importance of a target in the network. As shown in Figure 4(b), the darker color indicates a higher degree. The analysis results showed that IL-6 (degree=29), CASP3 $($ degree $=26)$, VEGFA $($ degree $=24)$, MYC $($ degree $=23)$, EGFR (degree $=23)$, ESR1 $($ degree $=22)$, and ERBB2 (degree $=21)$ were the pivotal targets in this network.

3.5. GO and Pathway Enrichment Analyses. We carried out GO enrichment analyses to further determine the functions of these shared targets from three aspects. As shown in Figure 5(a), twenty-six functional terms were enriched in the biological process category, such as cellular process, metabolic process, response to stimulus, and biological regulation. Fourteen functional terms were enriched in the cellular components category, such as cell part and organelle part. Additionally, nine functional terms were enriched in the molecular function category, such as binding, catalytic activity, and molecular function regulator.

The pathways analysis results revealed that 33 of 34 targets were found in the Reactome database, through which 319 pathways were identified (Figure 5(b)). The enriched pathways with a $P$ value $\leq 0.05$ were mainly concentrated in three categories: the immune system (26/33), signal transduction (29/33), and gene expression (transcription) (22/ 33). Moreover, 5 remarkable leading pathways with a $P$ value $\leq 0.001$ are described in Table 3 , as corrected by the false discovery rate (FDR), including the IL-4 and IL-13 signaling pathways; the ESR-mediated signaling pathway; the pathways of TFAP2 (AP-2) family regulating transcription of growth factors and their receptors; the extranuclear estrogen signaling pathway; and the nuclear receptors signaling pathway. 


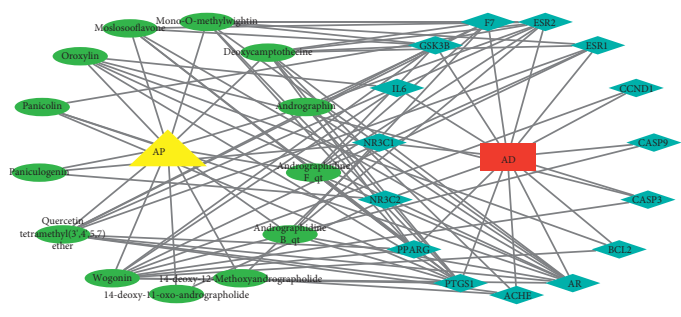

(a)

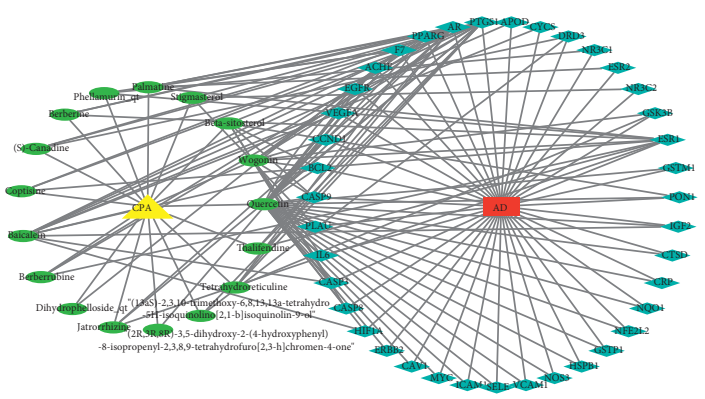

(c)

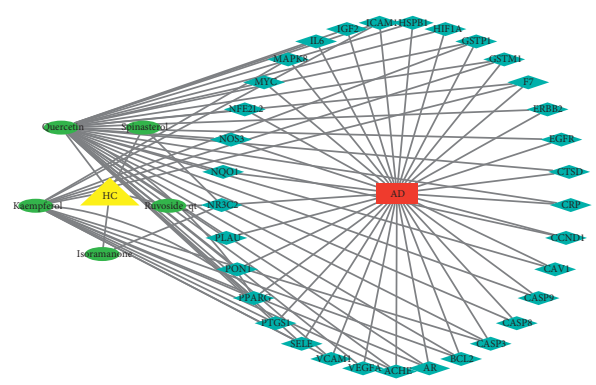

(e)

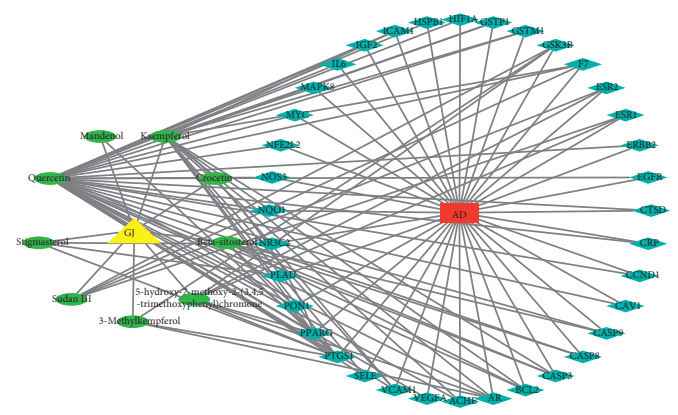

(g)

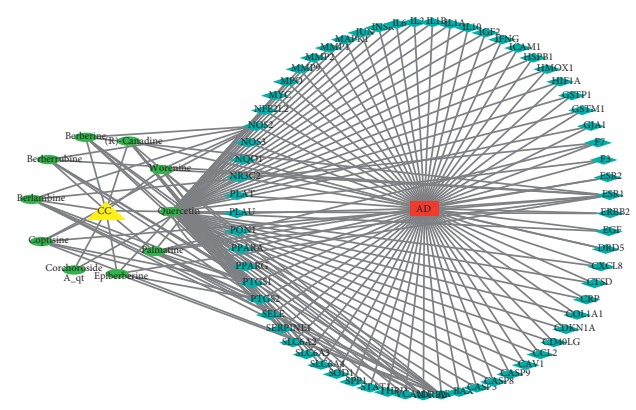

(b)

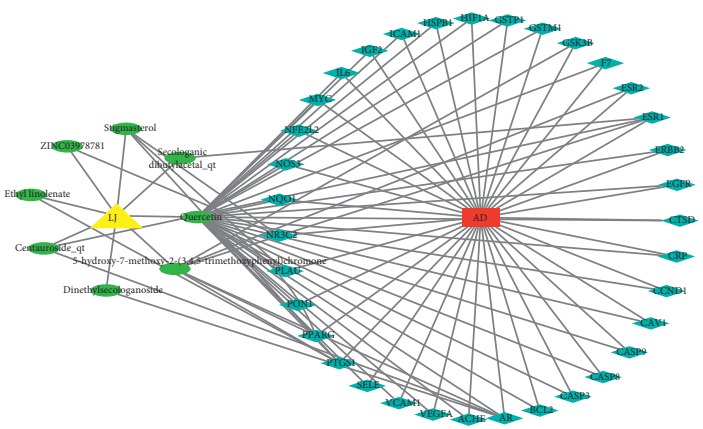

(d)

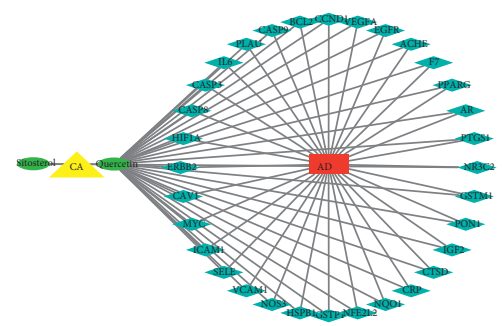

(f)

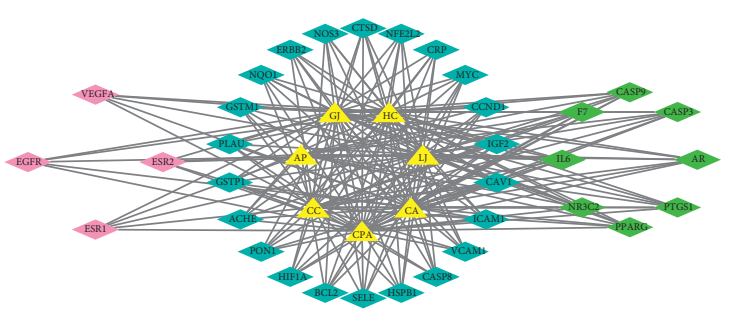

(h)

Figure 3: Network construction for anti-AD targets of 7 herbs. The herb-ingredient-target-disease networks of (a) AP, (b) CC, (c) CPA, (d) LJ, (e) HC, (f) CA, and (g) GJ. (h) Network of 34 shared targets for at least 5 herbs and AD (triangle: herbs; diamond: targets; purple: $n=5$; blue: $n=6$; green: $n=7$ ).

\section{Discussion}

$\mathrm{AD}$ is a typical neurodegenerative disease that seriously threatens human health worldwide. It is important to find new therapeutic drugs and treatment strategies for AD. In the past few decades, TCM has shown neuroprotective potential for treating AD owing to the synergistic effects of its multiple ingredients and targets [39]. HDTCM, one of the important components of TCM, has also been proven to be effective in the treatment of $\mathrm{AD}$. Thus, HDTCM is expected to open a new avenue for developing drugs for the treatment of AD. Although the anti-AD efficacy of some herbs of HDTCM was confirmed, the specific mechanism had not been elucidated. Accordingly, network pharmacology was used to study the analogous mechanism of the 7 herbs of HDTCM on AD, which will improve the utilization and development of HDTCM. 
TABLE 2: Information about ingredients and targets of 7 herbs.

\begin{tabular}{|c|c|c|c|c|c|}
\hline Herbs & $\begin{array}{c}\text { Numbers of active } \\
\text { ingredients }\end{array}$ & $\begin{array}{c}\text { Numbers of } \\
\text { targets }\end{array}$ & $\begin{array}{l}\text { Shared } \\
\text { targets }\end{array}$ & High-degree ingredients & $\begin{array}{l}\text { High-degree } \\
\text { targets }\end{array}$ \\
\hline $\mathrm{AP}$ & 13 & 127 & 15 & $\begin{array}{c}\text { Wogonin } \\
\text { Deoxycamptothecine } \\
\text { Quercetin tetramethyl }\left(3^{\prime}, 4^{\prime}, 5,7\right) \text { ether }\end{array}$ & $\begin{array}{l}\text { PTGS1 } \\
\text { AR }\end{array}$ \\
\hline $\mathrm{CC}$ & 10 & 222 & 65 & Quercetin palmatine & $\begin{array}{l}\text { PTGS2 } \\
\text { AR } \\
\text { PTGS1 }\end{array}$ \\
\hline $\mathrm{CPA}$ & 17 & 229 & 39 & $\begin{array}{c}\text { Quercetin } \\
\text { Wogonin baicalein }\end{array}$ & $\begin{array}{l}\text { PTGS1 } \\
\text { AR } \\
\text { ESR1 }\end{array}$ \\
\hline $\mathrm{LJ}$ & 8 & 115 & 35 & $\begin{array}{c}\text { Quercetin } \\
\text { 5-Hydroxy-7-methoxy-2-(3,4,5- } \\
\text { trimethoxyphenyl) chromone }\end{array}$ & $\begin{array}{l}\text { PTGS1 } \\
\text { AR } \\
\text { HC }\end{array}$ \\
\hline $\mathrm{HC}$ & 5 & 119 & 33 & $\begin{array}{c}\text { Quercetin } \\
\text { Kaempferol }\end{array}$ & $\begin{array}{l}\text { NR3C2 } \\
\text { PTGS1 }\end{array}$ \\
\hline CA & 2 & 80 & 32 & Quercetin & $\begin{array}{l}\text { GSTM1 } \\
\text { PTGS1 } \\
\text { IGF2 }\end{array}$ \\
\hline GJ & 9 & 170 & 36 & $\begin{array}{c}\text { Quercetin } \\
\text { Kaempferol }\end{array}$ & $\begin{array}{l}\text { PTGS1 } \\
\text { PPARG }\end{array}$ \\
\hline
\end{tabular}

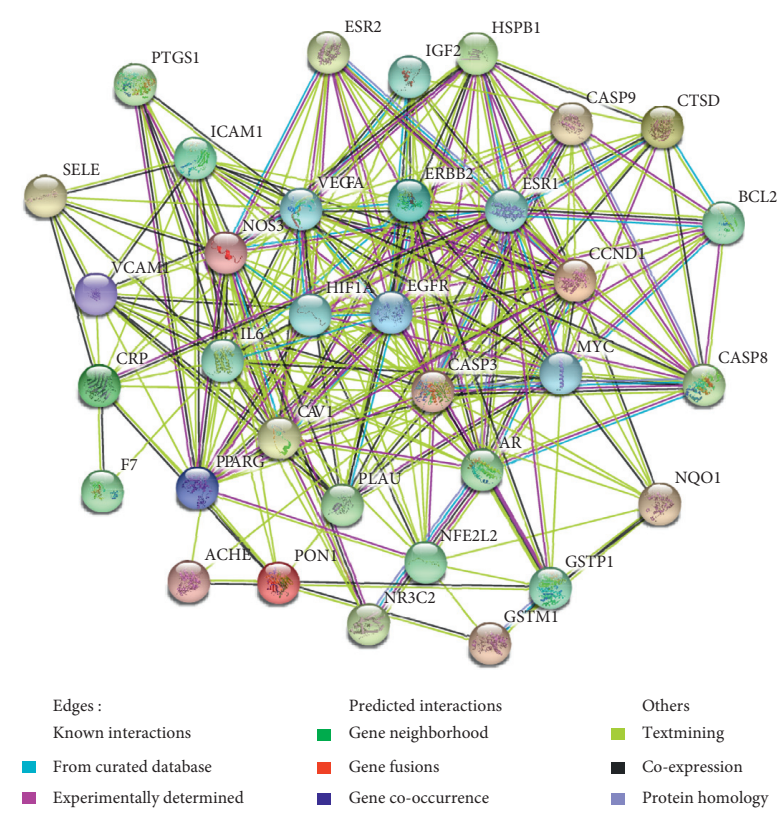

(a)

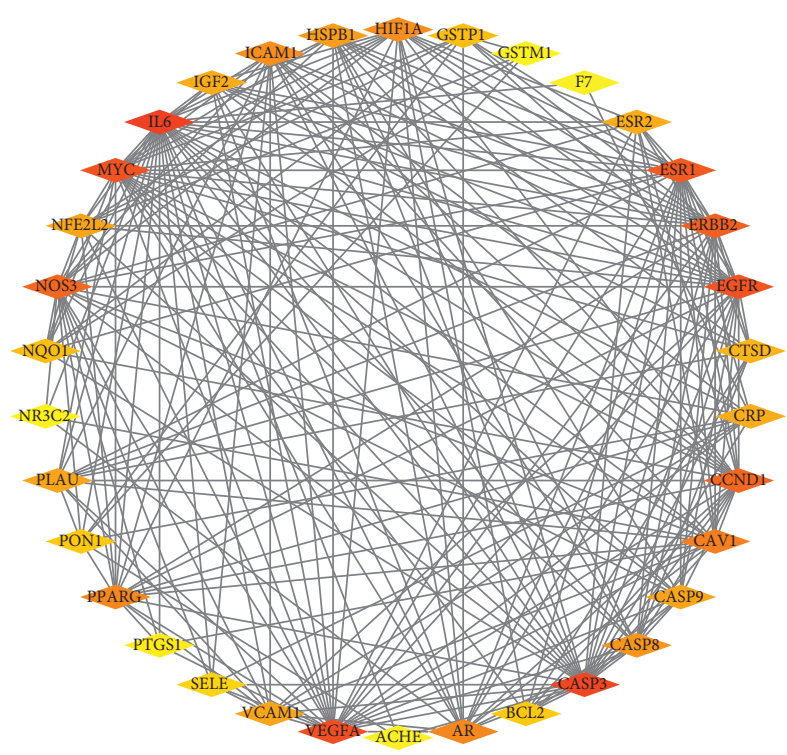

(b)

Figure 4: PPI network construction. (a) Nodes represent relevant targets; edges represent different interactions. (b) The darker the color, the higher the degree.

In our study, 7 herbs were ultimately screened for the network pharmacology analysis. A total of 64 active ingredients, 13 in AP, 10 in CC, 17 in CPA, 8 in LJ, 5 in HC, 2 in CA, and 9 in GJ, associated with 1032 targets, 111 in AP, 222 in CC, 219 in CPA, 113 in LJ, 119 in HC, 80 in CA, and 168 in GJ, were collected to construct herb-ingredient-target-disease networks. It was found that PTGS1 (Prostaglandin Endoperoxide Synthase 1) was the high-degree target in each of the networks. The results of one analysis showed that PTGS1 is associated with arachidonic acid- induced inflammation [40]. Moreover, researchers have confirmed that the arachidonic acid metabolism pathway mediates the development of $\mathrm{AD}$ [41] and $\mathrm{A} \beta$ plaques generation [42], and the upregulation of arachidonic acid has also been observed in an AD model [43].

Furthermore, by analyzing the PPI network constructed with 34 shared targets that were regulated by these herbs, we found that IL-6 (interleukin-6), CASP3 (caspase-3), and VEGFA (vascular endothelial growth factor) were the key gene targets with the highest calculated degree. In one study, 


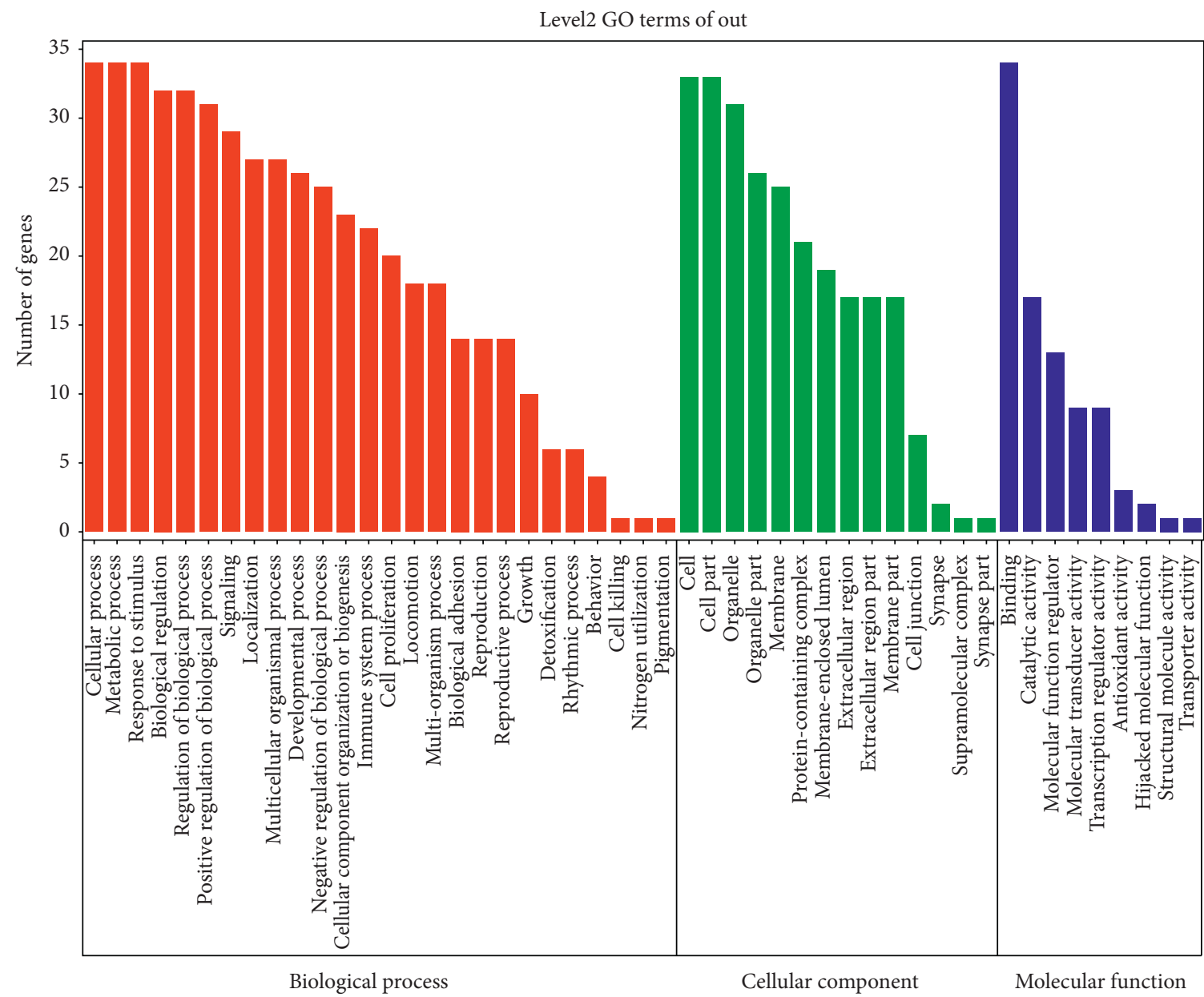

Biological process

Cellular component

Molecular function

(a)

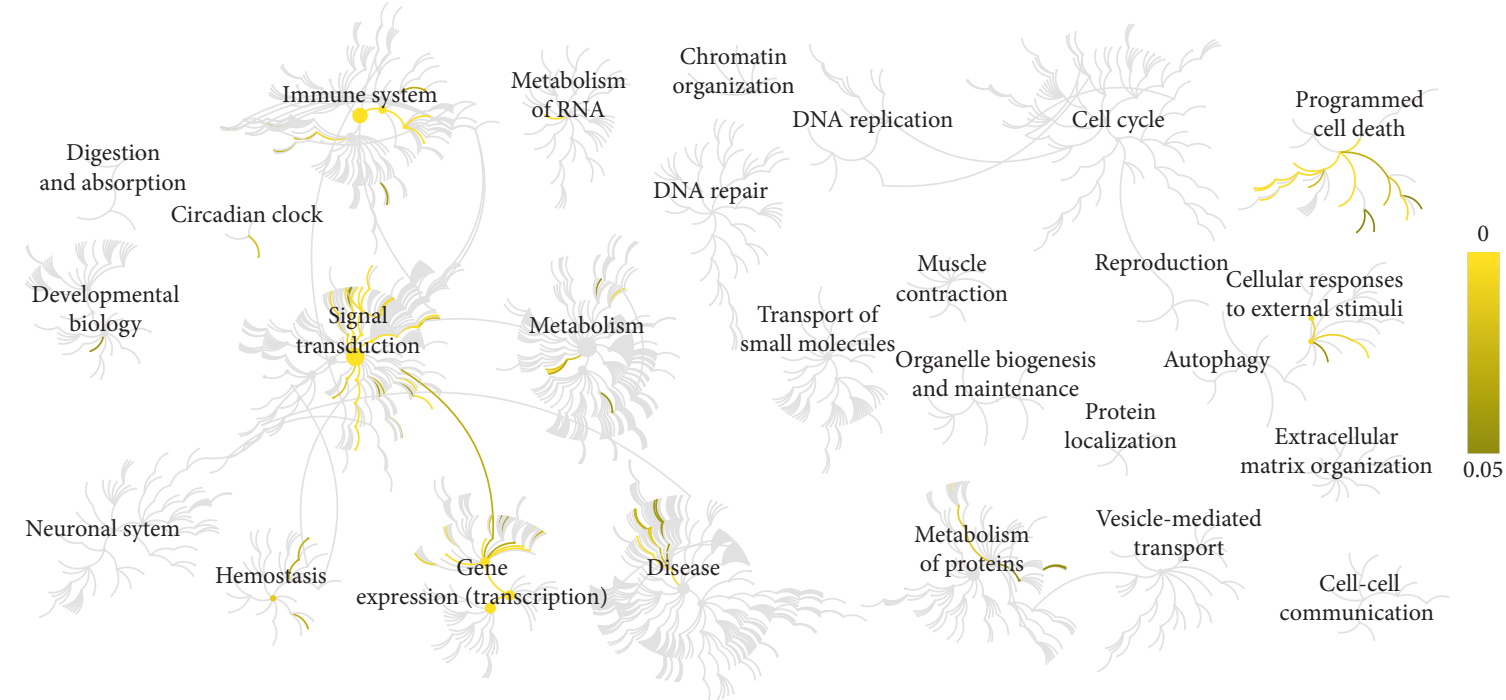

(b)

FIGURE 5: GO and pathway enrichment analysis. (a) GO analysis results of 34 common targets. (b) Reactome pathways analysis results of 34 common targets. 
TABle 3: Top 5 of significant pathways.

\begin{tabular}{|c|c|c|c|}
\hline Pathway name & $P$ value & FDR & Key genes \\
\hline IL- 4 and IL-13 signaling & $2.44 E-15$ & $7.91 E-13$ & $\begin{array}{l}\text { IL6; VCAM1; CCND1; MYC; BCL2; HIF1A; } \\
\text { ICAM1; VEGFA }\end{array}$ \\
\hline ESR-mediated signaling & $4.41 E-14$ & $7.14 E-12$ & $\begin{array}{l}\text { CCND1; NOS3; CAV1; MYC; BCL2; HSPB1; } \\
\text { CTSD; ESR1; ESR2; EGFR }\end{array}$ \\
\hline $\begin{array}{l}\text { TFAP2 (AP-2) family which regulates transcription of growth } \\
\text { factors and their receptors }\end{array}$ & $1.24 E-13$ & $1.16 E-11$ & ERBB2; ESR1; EGFR; VEGFA \\
\hline Extranuclear estrogen signaling & $1.44 E-13$ & $1.16 E-11$ & $\begin{array}{c}\text { CCND1; NOS3; CAV1; BCL2; HSPB1; ESR1; } \\
\text { ESR2; EGFR }\end{array}$ \\
\hline Nuclear receptors signaling & $1.16 E-12$ & $7.44 E-11$ & $\begin{array}{l}\text { CCND1; NOS3; CAV1; MYC; BCL2; HSPB1; } \\
\text { CTSD; ESR1; ESR2; EGFR }\end{array}$ \\
\hline
\end{tabular}

scientists reported that the level of IL-6, an inflammatory factor closely related to inflammation, in $\mathrm{AD}$ patients was significantly elevated [44]. Investigators have also found that downgrading the expression of CASP3 could reduce the inflammation induced by LPS in astrocytes associated with neurotic plaques in $\mathrm{AD}[45,46]$. In addition, one study found that VEGFA, an instigator of inflammation, was involved in the therapeutic regulatory network of $\mathrm{AD}$ and had a neuroprotective function [47].

Subsequently, the pathway analysis results showed that 34 targets were mainly enriched in pathways such as the IL-4 and IL-13 signaling pathway, the ESR-mediated signaling pathway, and the extranuclear estrogen signaling pathway. It was previously found that the IL-4 and IL-13 signaling pathways play a neuromodulating role by regulating the oxidative stress in $\mathrm{AD}$ and other neurodegenerative diseases [48]. Remarkably, IL-4 and IL-13 can activate microglia, the pivotal sources of inflammatory factors and oxidative stress in the brain, to induce $\mathrm{A} \beta$ degradation and improve cognitive impairment [49]. As implicated by increasing evidence, the regulation of neuroinflammation, one of the important incentives of $\mathrm{AD}$, is a vital therapeutic strategy. Simultaneously, researchers found that the estrogen receptor can provide a protective anti-inflammatory effect by inhibiting inflammation in neurodegenerative disorders such as $\mathrm{AD}$ and reducing $\mathrm{A} \beta$ deposition in the hippocampus to improve memory capacity $[50,51]$.

Taken together, these results indicate that the homologous anti-AD mechanism of the 7 herbs of HDTCM may play an impotant anti-neuroinflammatory role. However, there are some factors affecting the reliability of the results, including the differences in the databases, ingredient-screening indicators, and analysis tools. Consequently, specific experimental verification is an important part of further research.

CGA has multiple biological effects as an antioxidant, antiviral, and anticarcinogenic agent. Moreover, given that CGA can pass through the blood-brain barrier and play a direct role in the central nervous system [52], new CGA studies have paid more attention to its neuroprotective effect. For example, CGA can exert antiamnesic activity by inhibiting the expression of acetylcholinesterase and malondialdehyde [53]. In addition, investigators proved the neuroprotective effects of CGA in rat cerebellar granule neurons [54]. In another interesting study, researchers found that CGA combined with selenium nanoparticles inhibited $A \beta$ aggregation [55]. Both of these reports indicated that CGA had potential neuroprotective effects. Moreover, based on its anti-inflammatory effects, CGA is likely to be one of the main active ingredients against $\mathrm{AD}$ in the 7 herbs.

\section{Conclusion}

This study provides predictive insight into the mechanism of 7 frequently used herbs of HDTCM. The possible analogous anti-AD mechanism of the 7 herbs of HDTCM is related to anti-inflammation. Finally, we propose a hypothesis suggesting that CGA and quercetin, two main shared active ingredients, may play an indispensable role in the anti-AD efficacy of these 7 herbs, which requires verification and deserves further study.

\section{Data Availability}

The data used to support the findings of this study are available from the corresponding author upon request.

\section{Conflicts of Interest}

The authors declare that there are no conflicts of interest.

\section{Authors' Contributions}

QL and XZ designed the study and guided the experiment. HL conducted experiments and wrote manuscript. The data were analyzed by HL and LG. NW, LZ, and JL revised the paper. All authors read and approved the final manuscript.

\section{Acknowledgments}

This study was financially supported by the Science Technology Department of Zhejiang (No. 2017C37162) and Key Laboratory of Neuropsychiatric Drug Research of Zhejiang Province (No. 2019E10021).

\section{Supplementary Materials}

Initial screening of 12 herbs (Table S1). The information about ingredients and targets of Andrographis paniculate (AP), Coptis chinensis (CC), Cortex Phellodendri amurensis (CPA), Lonicera japonica (LJ), Houttuynia cordata (HC), Centella asiatica (CA), and Gardenia jasminoides (GJ) 
(Tables S2-S8). Target information of AD (Table S9). 34 shared targets for at least 5 herbs and AD (Table S10). (Supplementary Materials)

\section{References}

[1] M. Bothwell and E. Giniger, "Alzheimer's disease," Cell, vol. 102, no. 3, pp. 271-273, 2000.

[2] J.-L. Huang, Z.-H. Xu, S.-M. Yang et al., "Identification of differentially expressed profiles of Alzheimer's disease associated circular RNAs in a panax notoginseng saponins-treated Alzheimer's disease mouse model," Computational and Structural Biotechnology Journal, vol. 16, pp. 523-531, 2018.

[3] B. T. Hyman, C. H. Phelps, T. G. Beach et al., "National Institute on Aging-Alzheimer's Association guidelines for the neuropathologic assessment of Alzheimer's disease," Alzheimer's \& Dementia, vol. 8, no. 1, pp. 1-13, 2012.

[4] H. Cai, Y. Luo, X. Yan et al., "The mechanisms of BushenYizhi formula as a therapeutic agent against Alzheimer's disease," Scientific Reports, vol. 8, no. 1, 2018.

[5] E. Dolgin, "How to defeat dementia," Nature, vol. 539, no. 7628 , pp. 156-158, 2016.

[6] Y.-F. Zhang, Y. Huang, Y.-H. Ni, and Z.-M. Xu, "Systematic elucidation of the mechanism of geraniol via network pharmacology," Drug Design, Development and Therapy, vol. 13, pp. 1069-1075, 2019.

[7] F. Tang, Q. Zhang, Z. Nie, S. Yao, and B. Chen, "Sample preparation for analyzing traditional Chinese medicines," TrAC Trends in Analytical Chemistry, vol. 28, no. 11, pp. 1253-1262, 2009.

[8] J. Zhang, C. Yang, D. Wei et al., "Long-term efficacy of Chinese medicine Bushen Capsule on cognition and brain activity in patients with amnestic mild cognitive impairment," Pharmacological Research, vol. 146, p. 104319, 2019.

[9] S. S. Durairajan, Y. Y. Huang, P. Y. Yuen et al., "Effects of Huanglian-Jie-Du-Tang and its modified formula on the modulation of amyloid-beta precursor protein processing in Alzheimer's disease models," PLoS One, vol. 9, no. 7, Article ID e102923, 2014.

[10] Z. Zhang, X. Wang, D. Zhang, Y. Liu, and L. Li, “Geniposidemediated protection against amyloid deposition and behavioral impairment correlates with downregulation of mTOR signaling and enhanced autophagy in a mouse model of Alzheimer's disease," Aging, vol. 11, no. 2, pp. 536-548, 2019.

[11] F. G. Serrano, C. Tapia-Rojas, F. J. Carvajal, J. Hancke, W. Cerpa, and N. C. Inestrosa, "Andrographolide reduces cognitive impairment in young and mature A $\beta$ PPswe/PS-1 mice," Molecular Neurodegeneration, vol. 9, no. 1, p. 61, 2014.

[12] S. S. K. Durairajan, L.-F. Liu, J.-H. Lu et al., "Berberine ameliorates $\beta$-amyloid pathology, gliosis, and cognitive impairment in an Alzheimer's disease transgenic mouse model," Neurobiology of Aging, vol. 33, no. 12, pp. 2903-2919, 2012.

[13] M. D. d. S. Antos, M. C. A. Lmeida, N. P. L. Opes, and G. E. P. de Souza, "Evaluation of the anti-inflammatory, analgesic and antipyretic activities of the natural polyphenol chlorogenic acid," Biological \& Pharmaceutical Bulletin, vol. 29, no. 11, pp. 2236-2240, 2006.

[14] L. Gao, K. X. Wang, Y. Z. Zhou, J. S. Fang, X. M. Qin, and G. H. Du, "Uncovering the anticancer mechanism of Compound Kushen Injection against HCC by integrating quantitative analysis, network analysis and experimental validation," Scientific Reports, vol. 8, no. 1, p. 624, 2018.

[15] G. B. Zhang, Q. Y. Li, Q. L. Chen, and S. B. Su, "Network pharmacology: a new approach for Chinese herbal medicine research," Evid Based Complementary Alternative Medicine, vol. 2013, pp. 1-9, 2013.

[16] J. Ru, P. Li, J. Wang et al., "TCMSP: a database of systems pharmacology for drug discovery from herbal medicines," Journal of Cheminformatics, vol. 6, no. 1, 2014.

[17] W. Hu, W. Fu, X. Wei, Y. Yang, C. Lu, and Z. Liu, "A network pharmacology study on the active ingredients and potential targets of Tripterygium wilfordii hook for treatment of rheumatoid arthritis," Evidence Based Complementary Alternative Medicine, vol. 2019, Article ID 5276865, 2019.

[18] A. S. P. Pereira, M. J. Bester, and Z. Apostolides, "Exploring the anti-proliferative activity of Pelargonium sidoides DC with in silico target identification and network pharmacology," Molecular Diversity, vol. 21, no. 4, pp. 809-820, 2017.

[19] W. Wang, T. Liu, L. Yang et al., "Study on the multi-targets mechanism of triphala on cardio-cerebral vascular diseases based on network pharmacology," Biomedicine \& Pharmacotherapy, vol. 116, Article ID 108994, 2019.

[20] X. Xu, W. Zhang, C. Huang et al., "A novel chemometric method for the prediction of human oral bioavailability," International Journal of Molecular Sciences, vol. 13, no. 6, pp. 6964-6982, 2012.

[21] W. Tao, X. Xu, X. Wang et al., "Network pharmacology-based prediction of the active ingredients and potential targets of Chinese herbal Radix Curcumae formula for application to cardiovascular disease," Journal of Ethnopharmacology, vol. 145, no. 1, pp. 1-10, 2013.

[22] Y. Tao, K. Tian, J. Chen et al., "Network pharmacology-based prediction of the active compounds, potential targets, and signaling pathways involved in danshiliuhao granule for treatment of liver fibrosis," Evidence-Based Complementary and Alternative Medicine, vol. 2019, Article ID 2630357, , 2019.

[23] The UniProt Consortium, "UniProt: a worldwide hub of protein knowledge," Nucleic Acids Research, vol. 47, no. D1, pp. D506-D515, 2019.

[24] M. Safran, I. Dalah, J. Alexander et al., "GeneCards Version 3: the human gene integrator," Database, vol. 2010, Article ID baq020, 2010.

[25] D. Szklarczyk, A. L. Gable, D. Lyon et al., "STRING v11: protein-protein association networks with increased coverage, supporting functional discovery in genome-wide experimental datasets," Nucleic Acids Research, vol. 47, no. D1, pp. D607-D613, 2019.

[26] C. T. Lopes, M. Franz, F. Kazi, S. L. Donaldson, Q. Morris, and G. D. Bader, "Cytoscape Web: an interactive web-based network browser," Bioinformatics, vol. 26, no. 18, pp. 2347-2348, 2010.

[27] The Gene Ontology Consortium, "Expansion of the gene ontology knowledgebase and resources," Nucleic Acids Research, vol. 45, no. D1, pp. D331-D338, 2017.

[28] A. Fabregat, K. Sidiropoulos, P. Garapati et al., "The reactome pathway knowledgebase," Nucleic Acids Research, vol. 44, no. D1, pp. D481-D487, 2016.

[29] J. Y. Seo, E. Pyo, J. P. An, J. Kim, S. H. Sung, and W. K. Oh, "Andrographolide activates keap1/nrf2/ARE/HO-1 pathway in HT22 cells and suppresses microglial activation by Abeta42 through nrf2-related inflammatory response," Mediators Inflammation, vol. 2017, pp. 1-12, 2017.

[30] R. Yang, S. Liu, J. Zhou, S. Bu, and J. Zhang, "Andrographolide attenuates microglia-mediated $A \beta$ neurotoxicity partially through inhibiting NF- $\kappa B$ and JNK MAPK signaling pathway," Immunopharmacology and Immunotoxicology, vol. 39, no. 5, pp. 276-284, 2017. 
[31] Y. Li, S. Guan, C. Liu et al., "Neuroprotective effects of Coptis chinensis Franch polysaccharide on amyloid-beta $(A \beta)$-induced toxicity in a transgenic Caenorhabditis elegans model of Alzheimer's disease (AD)," International Journal of Biological Macromolecules, vol. 113, pp. 991-995, 2018.

[32] Y. Li, B. Wang, C. Liu et al., "Inhibiting c-Jun N-terminal kinase (JNK)-mediated apoptotic signaling pathway in PC12 cells by a polysaccharide (CCP) from Coptis chinensis against Amyloid- $\beta$ (A $\beta$ )-induced neurotoxicity," International Journal of Biological Macromolecules, vol. 134, pp. 565-574, 2019.

[33] Y.-F. Xian, Z.-X. Lin, S.-P. Ip, Z.-R. Su, J.-N. Chen, and X.-P. Lai, "Comparison the neuropreotective effect of Cortex Phellodendri chinensis and Cortex Phellodendri amurensis against beta-amyloid-induced neurotoxicity in PC12 cells," Phytomedicine, vol. 20, no. 2, pp. 187-193, 2013.

[34] S. Park, S. Kang, D. S. Kim, and B. R. Moon, “Agrimonia pilosa Ledeb., Cinnamomum cassia Blume, and Lonicera japonica Thunb. protect against cognitive dysfunction and energy and glucose dysregulation by reducing neuroinflammation and hippocampal insulin resistance in $\beta$-amyloid-infused rats," Nutritional Neuroscience, vol. 20, no. 2, pp. 77-88, 2017.

[35] H. Park and M. Oh, "Houttuyniae Herba protects rat primary cortical cells from $\mathrm{A} \beta 25-35$-induced neurotoxicity via regulation of calcium influx and mitochondria-mediated apoptosis," Human \& Experimental Toxicology, vol. 31, no. 7, pp. 698-709, 2012.

[36] M. Dhanasekaran, L. A. Holcomb, A. R. Hitt et al., "Centella asiaticaextract selectively decreases amyloid $\beta$ levels in hippocampus of Alzheimer's disease animal model," Phytotherapy Research, vol. 23, no. 1, pp. 14-19, 2009.

[37] N. E. Gray, J. A. Zweig, M. Caruso et al., "Centella asiatica attenuates hippocampal mitochondrial dysfunction and improves memory and executive function in $\beta$-amyloid overexpressing mice," Molecular and Cellular Neuroscience, vol. 93, pp. 1-9, 2018.

[38] C.-X. Zang, X.-Q. Bao, L. Li et al., "The protective effects of Gardenia jasminoides (fructus Gardenia) on amyloid- $\beta$-induced mouse cognitive impairment and neurotoxicity," The American Journal of Chinese Medicine, vol. 46, no. 2, pp. 389-405, 2018.

[39] S. G. Sreenivasmurthy, J. Y. Liu, J. X. Song et al., "Neurogenic traditional Chinese medicine as a promising strategy for the treatment of Alzheimer's disease," International Journal of Molecular Sciences, vol. 18, no. 2, 2017.

[40] R. Langenbach, S. G. Morham, H. F. Tiano et al., "Prostaglandin synthase 1 gene disruption in mice reduces arachidonic acid-induced inflammation and indomethacin-induced gastric ulceration," Cell, vol. 83, no. 3, pp. 483-492, 1995.

[41] H.-L. Gao, A.-H. Zhang, J.-B. Yu et al., "High-throughput lipidomics characterize key lipid molecules as potential therapeutic targets of Kaixinsan protects against Alzheimer's disease in APP/PS1 transgenic mice," Journal of Chromatography B, vol. 1092, pp. 286-295, 2018.

[42] W. Michno, P. M. Wehrli, H. Zetterberg, K. Blennow, and J. Hanrieder, "GM1 locates to mature amyloid structures implicating a prominent role for glycolipid-protein interactions in Alzheimer pathology," Biochimica et Biophysica Acta (BBA) - Proteins and Proteomics, vol. 1867, no. 5, pp. 458-467, 2019.

[43] J. G. Villamil-Ortiz and G. P. Cardona-Gomez, "cPLA2 and desaturases underlie the tau hyperphosphorylation offset induced by BACE knock-down in neuronal primary cultures," Biochimica et Biophysica Acta (BBA) - Molecular Basis of Disease, vol. 1864, no. 11, pp. 3696-3707, 2018.
[44] M. Gubandru, D. Margina, C. Tsitsimpikou et al., "Alzheimer's disease treated patients showed different patterns for oxidative stress and inflammation markers," Food and Chemical Toxicology, vol. 61, pp. 209-214, 2013.

[45] A. Gomez-Arboledas, J. C. Davila, E. Sanchez-Mejias et al., "Phagocytic clearance of presynaptic dystrophies by reactive astrocytes in Alzheimer's disease," Glia, vol. 66, no. 3, pp. 637-653, 2018.

[46] X.-H. Wang, Q. Liu, and Z.-T. Shao, "Deletion of JDP2 improves neurological outcomes of traumatic brain injury (TBI) in mice: inactivation of Caspase-3," Biochemical and Biophysical Research Communications, vol. 504, no. 4, pp. 805-811, 2018.

[47] V. Lanke, S. T. R. Moolamalla, D. Roy, and P. K. Vinod, "Integrative analysis of hippocampus gene expression profiles identifies network alterations in aging and Alzheimer's disease," Frontiers Aging Neuroscience, vol. 10, p. 153, 2018.

[48] J. Y. Jeong, Y. C. Chung, and B. K. Jin, "Interleukin-4 and interleukin-13 exacerbate neurotoxicity of prothrombin kringle-2 in cortex in vivo via oxidative stress," International Journal of Molecular Sciences, vol. 20, no. 8, p. 1927, 2019.

[49] K. Kawahara, M. Suenobu, A. Yoshida et al., "Intracerebral microinjection of interleukin-4/interleukin-13 reduces $\beta$-amyloid accumulation in the ipsilateral side and improves cognitive deficits in young amyloid precursor protein 23 mice," Neuroscience, vol. 207, pp. 243-260, 2012.

[50] H. Park, J. D. McEachon, and J. A. Pollock, "Synthesis and characterization of hydrogen peroxide activated estrogen receptor beta ligands," Bioorganic \& Medicinal Chemistry, vol. 27, no. 10, pp. 2075-2082, 2019.

[51] Z. Tian, J. Fan, Y. Zhao, S. Bi, L. Si, and Q. Liu, "Estrogen receptor beta treats Alzheimer's disease," Neural Regeneration Research, vol. 8, no. 5, pp. 420-426, 2013.

[52] H. Ito, X.-L. Sun, M. Watanabe, M. Okamoto, and T. Hatano, "Chlorogenic acid and its metabolitem-coumaric acid evoke neurite outgrowth in hippocampal neuronal cells," Bioscience, Biotechnology, and Biochemistry, vol. 72, no. 3, pp. 885-888, 2008.

[53] S.-H. Kwon, H.-K. Lee, J.-A. Kim et al., "Neuroprotective effects of chlorogenic acid on scopolamine-induced amnesia via anti-acetylcholinesterase and anti-oxidative activities in mice," European Journal of Pharmacology, vol. 649, no. 1-3, pp. $210-217,2010$.

[54] F. Taram, A. N. Winter, and D. A. Linseman, "Neuroprotection comparison of chlorogenic acid and its metabolites against mechanistically distinct cell death-inducing agents in cultured cerebellar granule neurons," Brain Research, vol. 1648, no. Pt A, pp. 69-80, 2016.

[55] L. Yang, N. Wang, and G. Zheng, "Enhanced effect of combining chlorogenic acid on selenium nanoparticles in inhibiting amyloid beta aggregation and reactive oxygen species formation in vitro," Nanoscale Research Letters, vol. 13, no. 1, p. 303, 2018. 